\title{
Not so critical appraisal of dapagliflozin
}

\section{Sheila A Doggrell}

School of Biomedical Science, Faculty of Health, Queensland University of Technology, Brisbane, QLD, Australia
Correspondence: Sheila A Doggrell School of Biomedical Science, Faculty of Health, Queensland University of Technology, GPO 2434, QLD 4002, Australia

Email sheila.doggrell@qut.edu.au
This article was published in the following Dove Press journal:

Patient Preference and Adherence

I8 August 2014

Number of times this article has been viewed

\section{Dear editor}

A recent review by Salvo et al published in Patient Preference and Adherence concerned dapagliflozin, and was titled "Patient considerations in the management of type 2 diabetes - critical appraisal of dapagliflozin". ${ }^{1}$ Having read the article, I do not consider it to be a critical appraisal of dapagliflozin. Thus, after comparing dapagliflozin with other oral antidiabetic medications, the authors concluded that "Dapagliflozin's noted blood pressure reduction, weight loss, and low potential to cause hypoglycemia are advantageous, when compared with currently available oral medications". ${ }^{1}$ This statement is not supported by the content of the review and/or the literature.

In their review of the efficacy of dapagliflozin, Salvo et al have provided little or no evidence that dapagliflozin reduces blood pressure. Dapagliflozin monotherapy was initially suggested to reduce blood pressure by a small amount at 24 weeks. ${ }^{2}$ However, the ability of dapagliflozin to reduce blood pressure is small, and is not always statistically significant. ${ }^{3,4}$ Thus, the reduction of blood pressure with dapagliflozin cannot be considered to be notable, and is unlikely to have any clinical significance.

Secondly, Salvo et al do provide some evidence of weight loss with dapagliflozin, of up to $4.65 \mathrm{~kg},{ }^{1}$ but do not critically appraise the significance of this. Although it is well established that being overweight or obese is a risk factor for diabetes and cardiovascular disease, it has not been shown that reducing weight in subjects with diabetes reduces long term cardiovascular outcomes. Thus, the Look AHEAD (Action for Health In Diabetes) trial in overweight or obese subjects with type 2 diabetes, compared an intensive lifestyle intervention to promote weight loss to diabetes support and education (control group). This trial was abandoned after 9.6 years, on the basis of a futility analysis for the primary outcome, which was a composite of death from cardiovascular causes, nonfatal myocardial infarction, nonfatal stroke, or hospitalization for angina. ${ }^{5}$ The futility in Look AHEAD was shown despite an initial 8.6\% body weight loss (from a baseline of approximately $101 \mathrm{~kg}$ ) and reduction in glycated hemoglobin of approximately $0.6 \%$ (from a baseline of about $7.3 \%$ ) in the intensive lifestyle intervention group. ${ }^{5}$ Given the findings of Look AHEAD, it is unlikely that a reduction in body weight of up to $4.65 \mathrm{~kg}$ with dapagliflozin is alone going to be beneficial in type 2 diabetes.

Also, Salvo et al suggest that the weight loss with dapagliflozin is an advantage over other oral medications in treating subjects with type 2 diabetes. ${ }^{1}$ Weight gain is observed with the sulfonylureas and the thiazolidinediones (eg, rosiglitazone). Thus, the weight loss with dapagliflozin may be an advantage over these medicines. However, the dipeptidylpeptidase inhibitors (gliptins) do not cause weight gain, and usually cause weight loss eg, saxagliptin. ${ }^{6}$ Glucagon-like peptide 1 receptor (GLP-1R) agonists, which are administered subcutaneously, also cause weight loss eg, exenatide. ${ }^{7}$ 
It seems to me, that these groups of anti-diabetic drugs, and their ability to cause weight loss, should have been part of any critical appraisal of dapagliflozin.

Thirdly, Salvo et al have suggested that dapagliflozin has a lesser ability to cause hypoglycemia than other oral antidiabetes medicines, and provided evidence that dapagliflozin has a low potential to cause hypoglycemia compared to the sulfonylureas (glipazide, glimepride), ${ }^{1}$ but not to other oral anti-diabetic medications. Thus, there is no evidence available to support dapagliflozin having lower hypoglycemia potential than metformin or GLP-1R agonists/gliptins, all of which have a lesser propensity to cause hypoglycemia than the sulfonylureas, eg, exenatide, ${ }^{7}$ saxagliptin. ${ }^{6}$ Also, definitive evidence for any low potential to cause hypoglycemia can only be obtained from comparative trials, and (at present) there are no published studies comparing metformin or the GLP-1R agonists/gliptins to dapagliflozin, let alone comparing their potential to cause hypoglycemia.

The authors did conclude that "long-term clinical trials and post-marketing studies are needed to further investigate dapagliflozin's cardiovascular profile, and its impact on morbidity and mortality", ${ }^{1}$ but this seems somewhat understated to me. To date, cardiovascular events with dapagliflozin have only been evaluated in subjects with type 2 diabetes, not having major cardiovascular disease. In their submission to the US Food and Drug Administration (FDA) about dapagliflozin, Bristol-Myers Squibb included a meta-analysis of cardiovascular events in their clinical program, in which only $37 \%$ of subjects had a history of cardiovascular disease (excluding hypertension). ${ }^{8}$ In this group, the cardiovascular events (cardiovascular death, stroke, myocardial infarction, hospitalization of unstable angina) were low; probably because the enrolled population was not high risk. ${ }^{8}$ In this low risk group, cardiovascular events occurred in $1.99 \%$ of the comparator group, compared to $1.64 \%$ per subject year in the dapagliflozin, and this was not significantly different. ${ }^{8}$

The multicenter trial that will eventually be able to evaluate the cardiovascular safety of dapagliflozin, is the effect of dapagliflozin on the incidence of cardiovascular events (DECLARE-TIMI58). ${ }^{9}$ DECLARE-TIMI58 is a Phase III trial recruiting 22,200 subjects with type 2 diabetes and high cardiovascular risk subjects. $^{9}$ In this trial, dapagliflozin or placebo will be added to the subjects' current anti-diabetes medicines, and the primary outcome measure is the composite of cardiovascular death, myocardial infarction or ischemic stroke in subjects with type 2 diabetes. ${ }^{9}$ DECLARE-TIMI58 is due to be completed in April 2019. ${ }^{9}$ By this time, it will be about 5 years since the FDA approved dapagliflozin for use in subjects with type 2 diabetes. Thus, dapagliflozin will be available for 5 years without proven cardiovascular efficacy or safety. It seems to me, that dapagliflozin should not have been registered, until DECLARE-TIMI58 was complete, and cardiovascular safety established. This point should have been considered by Salvo et al in a critical appraisal of dapagliflozin.

In conclusion, dapagliflozin does not have a noted ability to reduce blood pressure. However, dapagliflozin does cause weight loss and have a low potential to cause hypoglycemia, but this is not necessarily an advantage, as other medicines available for the treatment of type 2 diabetes also cause weight loss and have a low propensity to cause hypoglycemia. Most importantly, the cardiovascular clinical outcome study DECLARE-TIMI58 being undertaken with dapagliflozin will not report for 5 years, and thus dapagliflozin will be available for the treatment of type 2 diabetes for 5 years without proven cardiovascular efficacy or safety.

\section{Disclosure}

The author reports no conflicts of interest in this communication.

\section{References}

1. Salvo MC, Brooks AD, Thacker SM. Patient considerations in the management of type 2 diabetes - critical appraisal of dapagliflozin. Patient Pref Adherence. 2014;8:493-502.

2. Ferrannini E, Ramos SJ, Salsali A, Tang W, List JF. Dapagliflozin monotherapy in type 2 diabetic patients with inadequate glycemic control by diet and exercise - a randomized, double-blind, placebo-controlled, phase 2 trial. Diabetes Care. 2010;33:2217-2224.

3. Ji L, Ma J, Li H, et al. Dapagliflozin as monotherapy in drug-naïve Asian patients with type 2 diabetes mellitus: a randomized, blinded, prospective phase III study. Clin Ther. 2014;36:84-100.

4. Jabbour SA, Hardy E, Sugg J, Parikh S. Dapagliflozin is effective as add-on therapy to sitagliptin with or without metformin: a 24-week, multicentre, randomized, double-blind, placebo-controlled study. Diabetes Care. 2014;37:740-750.

5. Wing RR, Bolin P, Brancati FL, et al. for the Look AHEAD Research Group. Cardiovascular effects of intensive lifestyle intervention in type 2 diabetes. N Engl J Med. 2013;369:145-154.

6. Mintz ML, Minervini G. Saxagliptin versus glipizide as add-on therapy to metformin: assessment of hypoglycemia. Curr Med Res Opin. 2014;30:761-770.

7. Gallwitz B, Guzman J, Dotta F, et al. Exenatide twice daily versus glimepiride for prevention of glycaemic deterioration in patients with type 2 diabetes with metformin failure (EUREXA): an open-label randomised controlled trial. Lancet. 2012;379(9833):2270-2278.

8. Dapagliflozin (Forxiga). Summary of product characteristics, BristolMyers Squibb, Uxbridge, UK. 2014. Available from: http://www. medicines.org.uk/EMC/medicine/27188. Accessed May 26, 2014.

9. ClinicalTrials.gov. Multicenter trial to evaluate the effect of dapagliflozin on the incidence of cardiovascular events (DECLARE-TIMI58). Available from: http://clinicaltrials.gov/ct2/show/NCT01730534?term=dapa gliflozin\&rank=36. Accessed May 26, 2014. 


\section{Author's response}

Amie D Brooks

Department of Pharmacy Practice, St Louis College of Pharmacy, St Louis, MO, USA

Correspondence: Amie D Brooks

Department of Pharmacy Practice, St Louis College of Pharmacy, 4588

Parkview Place, St Louis, MO 631 I0, USA

Tel +l 3144468500

Fax +I 3I4 4468500

Email amie.brooks@stlcop.edu

\section{Dear editor}

In response to the letter by Doggrell regarding our recent publication entitled, "Patient considerations in the management of type 2 diabetes - critical appraisal of dapagliflozin", we would first like to express appreciation for the interest in our publication as well as the opportunity to respond. ${ }^{1}$ The overall intent of our review of dapagliflozin was to provide a critique of the available primary literature for this newly available medication (approved in the US during the time of manuscript preparation), however, we did not intend to be "critical" in the negative sense described by Doggrell. We contend that our review provides a fair and unbiased overview of the available primary literature and does not over or understate any of the findings. As mentioned by Doggrell, there is very limited comparative effectiveness research with dapagliflozin, and within diabetes therapies in general. The intention of the publication was not to "compare" available therapies but rather to present the available evidence for dapagliflozin and succinctly summarize the advantages and disadvantages of this compound, in the context of other therapies, with special attention to patient considerations. This is similar to the approach taken by the American Diabetes Association (ADA) and European Association for the Study of Diabetes (EASD) consensus algorithm which advises clinicians to consider second line therapies (following metformin) based upon risk of hypoglycemia, weight gain, cost, and major side effects. ${ }^{2}$

In reference to blood pressure lowering potential, our review includes four studies that demonstrate blood pressure lowering with dapagliflozin $(-2.1$ to $-6.4 \mathrm{mmHg}$ systolic blood pressure and -1.7 to $-3.3 \mathrm{mmHg}$ diastolic blood pressure).$^{3-6} \mathrm{We}$ acknowledge that the clinical significance of this blood pressure reduction is unknown at this time given the limitations of blood pressure reduction as a surrogate for clinical outcomes and the differences observed in outcomes between antihypertensive medication classes. However, as with other patient considerations, note this as a potentially favorable characteristic for patients with concomitant diabetes and hypertension.

In regards to the weight loss (up to $4.65 \mathrm{~kg}$ ) demonstrated with dapagliflozin and the futility of the Look AHEAD study, we would like to acknowledge that there are benefits of weight loss other than cardiovascular outcomes and also comment on limitations of the Look AHEAD data. ${ }^{7}$ As previously mentioned, our article focuses on patient considerations in the management of type 2 diabetes. In working with thousands of overweight and obese patients over the past fifteen years our observation has been that there is patient preference for medications that contribute to weight loss. Beyond preference, the ADA EASD algorithm supports weight loss as a consideration in selecting second line therapy. ${ }^{2}$ In the Look AHEAD trial, the patient population had an average duration of diabetes of 14 years, by study-end the absolute difference in weight loss between intervention and control groups was around $2.5 \%$, and the overall cardiovascular event rate was much lower than anticipated. ${ }^{7}$ These factors likely contributed to the lack of cardiovascular benefit observed with the weight loss intervention in this study. Additionally, despite these limitations, patients in the intervention group demonstrated reductions in urinary incontinence, sleep apnea, and depression and improvements in quality of life, mobility, and physical functioning. ${ }^{7}$ Though cardiovascular outcomes were not improved, these non-cardiovascular benefits are certainly important patient considerations when selecting therapy.

We too look forward to the eventual availability of results from the DECLARE-TIMI58 trial and acknowledge that those findings will undoubtedly strengthen the understanding of dapagliflozin's cardiovascular impact and subsequent place in therapy. The United States Food and Drug Administration requires that new drug sponsors provide data supporting cardiovascular safety prior to the approval of any new therapy for type 2 diabetes. ${ }^{8}$ The FDA recommendations are to include patients in Phase II and III studies who have a longer duration of diabetes, are elderly, and have some degree of renal dysfunction. ${ }^{8}$ Dapagliflozin's available evidence includes patients with these characteristics and has demonstrated no increased cardiovascular risk. While it is certainly ideal to use medications with cardiovascular benefit (so far only metformin) and those with a long track record of cardiovascular safety; unfortunately, these options are currently very limited.

We maintain that dapagliflozin represents a promising new therapeutic approach to the treatment of type 2 diabetes that is a viable second line (or beyond) therapy option for select patients. 


\section{Disclosure}

The author reports no conflicts of interest in this communication.

\section{References}

1. Salvo MC, Brooks AD, Thacker SM. Patient considerations in the management of type 2 diabetes - critical appraisal of dapagliflozin. Patient Pref Adherence. 2014;8:493-502.

2. Inzucchi SE, Bergenstal RM, Buse JB, et al. Management of hyperglycemia in type 2 diabetes: a patient-centered approach: position statement of the American Diabetes Association (ADA) and European Association for the Study of Diabetes (EASD). Diabetes Care. 2012;35(6):1364-1379.

3. Ferrannini E, Ramos SJ, Salsali A, Tang W, List JF. Dapagliflozin in monotherapy in type 2 diabetes patients with inadequate glycemic control by diet and exercise: a randomized, double-blind, placebo-controlled, phase 3 trial. Diabetes Care. 2010;33(10):2217-2224.
4. Komoroski B, Vachharajani N, Boulton D, et al. Dapagliflozin, a novel SGLT2 inhibitor, induces dose-dependent glycosuria in healthy subjects. Clin Pharmacol Ther. 2009;85(5):520-526.

5. Wilding JP, Norwood P, T'joen C, et al. A study of dapagliflozin in patients with type 2 diabetes receiving high doses of insulin plus insulin sensitizers: applicability of a novel insulin-independent treatment. Diabetes Care. 2009;32(9):1656-1662.

6. List JF, Woo V, Morales E, Tang W, Fiedorek FT. Sodium-glucose cotransport inhibition with dapagliflozin in type 2 diabetes. Diabetes Care. 2009;32(4):650-657.

7. Look AHEAD Research Group, Wing RR, Bolin P, et al. Cardiovascular effects of intensive lifestyle intervention in type 2 diabetes. $N$ Engl J Med. 2013;369:145-154.

8. US Department of Health and Human Services Food and Drug Administration Center for Drug Evaluation and Research: Guidance for Industry. Diabetes Mellitus - Evaluating Cardiovascular Risk in New Antidiabetic Therapies to Treat Type 2 Diabetes. Available from: http:/www.fda.gov/ downloads/drugs/guidancecomplianceregulatoryinformation/guidances/ ucm071627.pdf. Accessed July 27, 2014.

\section{Publish your work in this journal}

Patient Preference and Adherence is an international, peer-reviewed, open access journal that focuses on the growing importance of patient preference and adherence throughout the therapeutic continuum. Patient satisfaction, acceptability, quality of life, compliance, persistence and their role in developing new therapeutic modalities and compounds to optimize clinical outcomes for existing disease states are major areas of interest for the journal. This journal has been accepted for indexing on PubMed Central. The manuscript management system is completely online and includes a very quick and fair peer-review system, which is all easy to use. Visit http://www dovepress.com/testimonials.php to read real quotes from published authors. 